\title{
The research of finely dispersed iron powder moistening applying the $\mathrm{pH}-$ metry method
}

\author{
Kseniya Ikonnikova ${ }^{1, *}$, Lyubov Ikonnikova $^{1}$, Ekaterina Koltunova $^{1}$, and Maxim Murashkin ${ }^{2}$ \\ ${ }^{1}$ Tomsk Polytechnic University, Tomsk, Russia \\ ${ }^{2}$ Institute of Physics of Advanced Materials, Ufa State Aviation Technical University, Ufa, Russian \\ Federation
}

\begin{abstract}
This research is aimed at solving crucial problem to diagnose and prognosticate moistening of fine-grained (finely-dispersed) materials. The article considers the dependence of surface moistening from the nature of the primary acid-base center. It demonstrates the advantages of acidbase property study of the surface applying the hydrolytic adsorption method. The research of hydrolytic adsorption on the ferric oxide with a haematite structure was carried out. Ferric oxide is presented in various states (massive and in the form of oxide film on the iron core of nanometer size). The identification of the composition and structure of the ferricoxide compound was carried out applying the method of transmission electron microscopy (TEM) and X-ray diffraction analysis (XRDA). The authors found out the identity of the behavior characteristics of hydrolytic adsorption on the haematite in various states. The paper also describes the inhibition mechanism of haematite moistening, which explains its hydrophobic behavior. The material of this article can be useful for its implementation in industrial, research and school laboratories.
\end{abstract}

\section{Introduction}

Finely dispersed metal powders are widely used in mechanical engineering, power engineering, medicine, construction, environmental protection and etc. If the process occurs in the system "solid body-liquid" then the efficiency of powder use depends on the compatibility of solid body and liquid. Therefore, it is very important to know such characteristic as surface moistening to solve the problem of obtaining products with desired functional properties.

Most of the methods used for quantitative assessment of moistening [1] are not appropriate for powders of nanometer size due to their high reactive capacity, small size and spherocity of particles. Therefore, the development of the methods used for quantitative assessment is very significant. These methods are based on the measurement of indirect values related to the moistening phenomenon. At qualitative level "moistening" is defined by opposite conceptions such as "hydrophilic behavior / hydrophobic behavior".

* Corresponding author: ikonna@yandex.ru 
Since the finely dispersed iron is pyrophoric, it is necessary to passivate it in advance in oxidizing medium. Whereby the oxide layer is formed on the surface of metal particles which phase compound depends on the passivating conditions [2-4]. The change of the oxidizing medium composition, temperature and curing period leads to the formation of nano-dimensional oxide layer which has a complex composition: $\mathrm{FeO}, \mathrm{Fe}_{2} \mathrm{O}_{3}$ and $\mathrm{Fe}_{3} \mathrm{O}_{4}$ of different modifications. These compounds have different acid-base surface centers in massive crystalline state and adsorb water through different mechanisms. The research of hydrolytic adsorption conducted earlier on $\mathrm{Fe}_{2} \mathrm{O}_{3}$ and $\mathrm{Fe}_{3} \mathrm{O}_{4}$ samples in massive state shows considerable differences of $\mathrm{pH}$ change in aqueous suspension [5-7]. Acidification and alkalization of aqueous suspension were observed, which can be related with the transition of reaction products in the medium $\left(\mathrm{H}^{+}\right.$or $\mathrm{OH}^{-}$-ions). The $\mathrm{pH}$ value corresponds with the mass transfer unit [8].

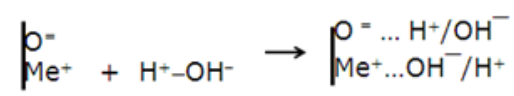

The analysis of the mass transfer speed ( $v p H=\Delta p H_{i} / \Delta \tau$, min ) with time allows us to establish the reason for inhibition process of moistening and assess the hydrophilic behavior/ hydrophobic behavior of the surface at qualitative level. The dimension of solid body particles significantly affects the absolute value of the indicators of the heterogeneous system. Therefore, the change of the mass transfer speed is only considered and does not require the consideration of the specific surface level.

All the above mentioned actualizes the study of moistening process of structures with nanometer dimension by means of hydrolytic adsorption method.

The aim of this work is to study the process of wetting in an aqueous medium of highly dispersed iron powder obtained by Electric Explosion of Wire method (EEW iron) and passivated in an oxidizing atmosphere.

\section{Experimental setup and methods}

The subject of inquiry is iron nanopowder («sample 1» - iron EEW) obtained applying the electric blasting method of a steel wire of the St 3 type (State Standard 380-2005) in the argon environment at gas pressure 0.2 megapascal [9]. The obtained powder was passivated by means of slow air inflow in the container with nanopowder over the period of 72 hours at $20^{\circ} \mathrm{C}$. This process was carried out in accordance with the recommendations [10]. The sample was obtained in the Institute of Physics of Strength and Material Science (RAS, Tomsk).

The composition and structure of the oxide film on the iron core were studied applying the method of transmission electron microscopy (TEM). The «JEOL JEM - 2100F» equipment of Nano-Center of TPU was used for this purpose.

The identification of comparison-sample («comparison-sample 2»- haematite in massive condition (state) was carried out applying X-ray diffraction analysis (XRDA) on the "Diffray -401 " diffractometer. To decrypt the $\mathrm{x}$-ray used $\mathrm{x}$ ray diffraction of ASTM and JCPDS card files.

The moistening of sample surface was studied by variation of $\mathrm{pH}$-metry methods of hydrolytic adsorption [11]. The initial water acidity was $6.7 \div 6.9 \mathrm{pH}$ units. The suspension was prepared in the ratio water /solid body $=50$. The change of $\mathrm{pH}$ of aqueous suspension over a period of time was registered by a special device of $\mathrm{pH}-150 \mathrm{M}$ type (measuring accuracy is $\pm 0.03 \mathrm{pH}$ units). The parameters were measured every 5 seconds of the contact by continuous stirring of aqueous suspension using a magnetic stirrer within 90 minutes. The obtained kinetic mass transfer curve $(\mathrm{pH}=\mathrm{f}(\mathrm{time}))$ was differentiated with various 
stepping time interval to get data about mass transfer $\left(\nu p H=\Delta p H_{i} / \Delta \tau, \min \right)$ and moistening rate.

\section{Results and discussion}

It was necessary to solve a range of tasks to achieve the desired objective:

1. To identify the phase composition of the oxide film formed on the EEW iron core during passivation.

2. To investigate the process of hydrolytic adsorption on the nano-dimensional EEW iron

3. To give qualitative assessment of moistening (hydrophilic behaviour / hydrophobic behavior) applying mathematical treatment of kinetic curves of hydrolytic adsorption.

\subsection{The identification of the phase composition of the oxide film formed on the iron core during passivation}

The first stage of the given work was to carry out investigation on identification of the phase composition of the sample oxide layer. It is related to the fact that even minor deviation from the production procedures results in considerable changes of the sample dispersion, oxide film composition and structure [2].

The images of nanoparticles are presented in Figures 1-3. The analysis of TEM images shows (Fig.1) that the particles are polydisperse, they are not agglomerated with each other, they have spherical shape and different dimension (size). The diameter of the body of large particles together with oxide film is $1000 \mathrm{~nm}$, whereas small particle diameter is only $50 \mathrm{~nm}$. The amount of large particles is small. The majority of particles are of different size from 60 till $200 \mathrm{~nm}$.

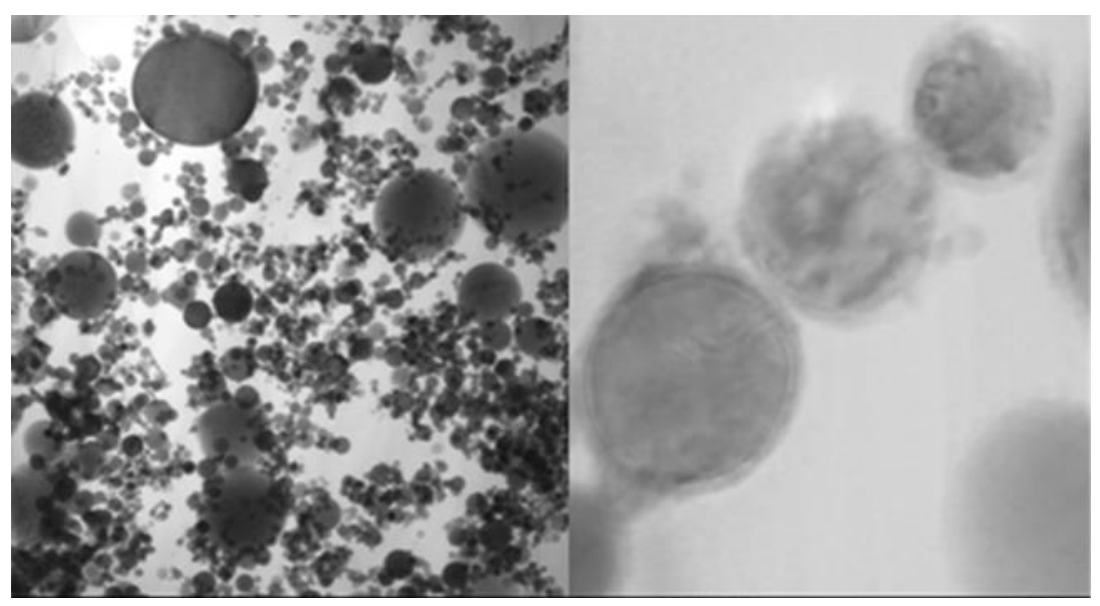

Fig.1. Microphotos with various expansion of electric blasting iron powder obtained applying the method of transmission electron microscopy (TEM).

Macrophoto (Fig. 3) allows defining the main characteristics of the particles of different size (Fig. 2). These characteristics are: oxide layer thickness, its continuity and crystallinity as well as the value of interplanar spacing. 


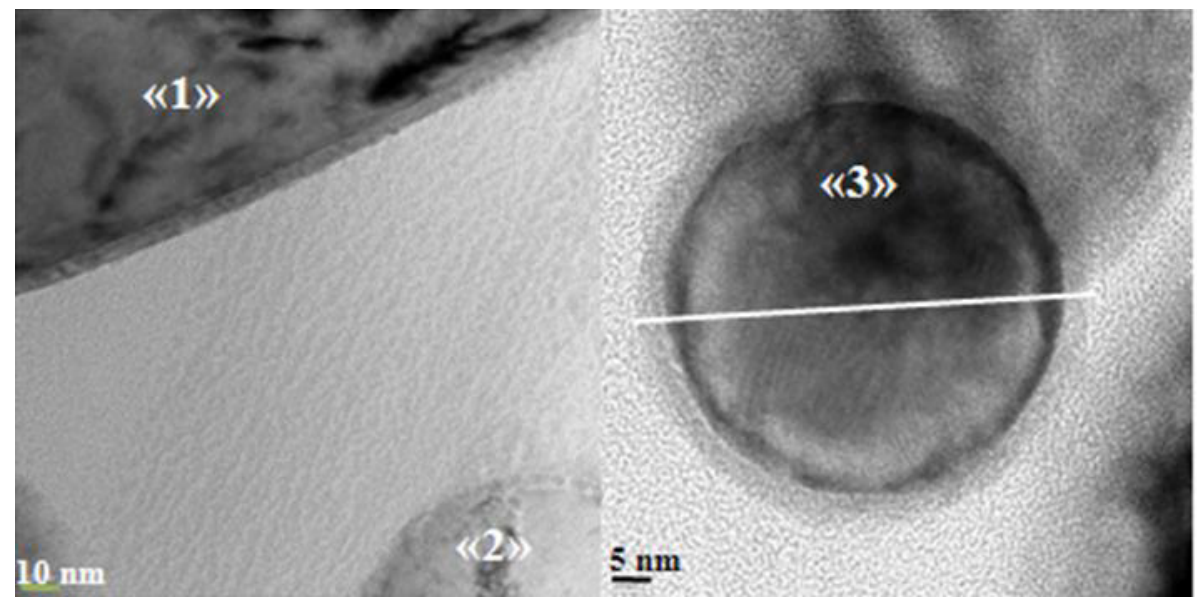

Fig. 2. Photo of TEM for particles of different size:

$\ll 1 »-(\mathrm{d}$ particles $=1000 \mathrm{~nm}), \quad\langle 2 »-(\mathrm{d}$ particles $=100 \mathrm{~nm}), \quad\langle 3 »-(\mathrm{d}$ particles $=70 \mathrm{~nm})$.

The TEM analysis showed (Fig.3) that the parameters of oxide layer for the particles with different dimension are almost identical:

- the thickness of oxide layer is uniform and equals $4 \div 5 \mathrm{~nm}$;

- the lines of atomic rows are uniform and have one direction. It testifies the single-layer coating and crystallinity of the oxide film;

- the distance between atomic rows is $0.269 \mathrm{~nm}$. It corresponds to the reflex with $\mathrm{d}(104)=0.269 \mathrm{~nm}$. This reflex has maximal intensity and is typical for ferric oxide with $\alpha-$ $\mathrm{Fe}_{2} \mathrm{O}_{3}$ structure (Fig.4). The absence of powder particles agglomeration is also a vivid evidence in the favor of $\alpha-\mathrm{Fe}_{2} \mathrm{O}_{3}$ formation, which magnetic susceptibility is low.

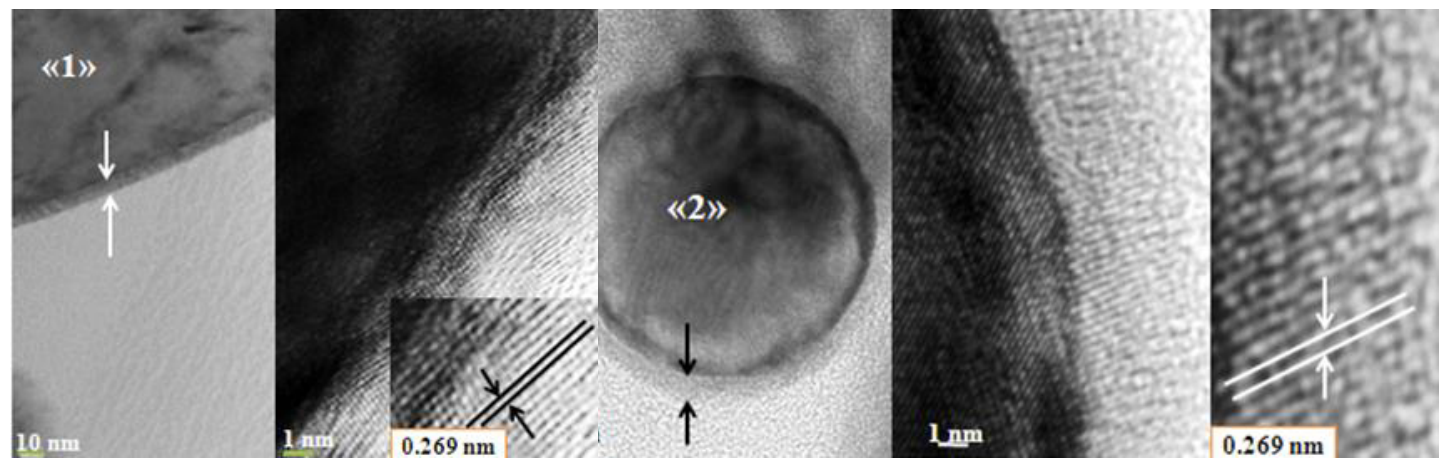

Fig. 3. The photo of TEM analysis of the oxide film of particles with different sizes (dimension): «1»- $(\mathrm{d}$ particles $=1000 \mathrm{~nm}), \quad « 2 »-(\mathrm{d}$ particles $=70 \mathrm{~nm})$.

\subsection{The research applying the method of hydrolytic adsorption}

The causes affecting the dimensional parameters and structure of low-dimensional particles are considered in monograph [2]. It is believed that one should be very attentive and careful with the idea of identifying phase composition of such structures by means of comparison between interatomic rows distances (defined by TEM method) and interplanar spacing of samples in massive state (defined applying X-ray diffraction analysis (XRDA)). 
One should be very attentive when there is reflection of one plane only. Confirmatory findings are required that were obtained by applying other methods.

The next stage of our research was the comparison of findings concerning the hydrolytic adsorption obtained for electric blasting iron powder ("sample 1") and ferric oxide with $\alpha$ $\mathrm{Fe}_{2} \mathrm{O}_{3}$ structure in massive state ("comparison- sample 2"). The identification of ferric oxide sample in massive state ("comparison- sample 2") was carried out applying X-ray diffraction analysis (XRDA). The findings of X-ray diffraction analysis show that the sample chosen for comparison is ferric oxide with $\alpha-\mathrm{Fe}_{2} \mathrm{O}_{3}$ structure (Fig. 4).

The findings of the hydrolytic adsorption research showed (Fig. 5) that being in contact for 20 minutes the kinetic mass transfer curves $(\mathrm{pH})$ have the same manner relative to the neutrality level and the kinetic curves of mass transfer speed $(\mathrm{v} \mathrm{pH})$ display the same mechanism (regularity) by speed gain and drop. It is vivid evidence that the film on the surface of passivated metallic iron has ferrioxide composition with $\alpha-\mathrm{Fe}_{2} \mathrm{O}_{3}$ structure. The rapid completion of speed instability (in two minutes after being in contact) is caused by the processes of external diffusion inhibition and characterizes the hydrophobic behavior of the surface. The explanation of external diffusion inhibition is as follows:

1. Primary active $\alpha-\mathrm{Fe}_{2} \mathrm{O}_{3}$ surface centers are $\mathrm{Fe}^{3+}$ cations which have octahedral coordination (arrangement) on oxygen. The density of the negative charge is high;

2. The adsorption of water molecules from air forms the secondary Lewes basic centers of brandsted type $\left(\mathrm{O}^{2} \ldots \mathrm{H}^{+} / \mathrm{OH}^{-}\right)$on the primary Lewes centers;

There is two-dimensional shielding film formed due to hydrogen bond between main brandsted centers. It offers resistance to the further moistening. The surface is hydrophobic [12].

\begin{tabular}{|l|l|l|l|l|l|}
\hline \multicolumn{3}{|c|}{ Sample } & \multicolumn{3}{c|}{$\begin{array}{c}\text { (PDF-11053) } \\
\text { Oxide. Fe } e_{2} \\
\text { Hematite. }\end{array}$} \\
\hline 2 teta & d (nm) & I $\%$ & 2 teta & d (nm) & I \\
\hline 26.40 & 0.424 & 3 & & - &. \\
\hline 30.31 & 0.370 & 22 & 30.50 & 0.368 & 18 \\
\hline 42.21 & 0.269 & 100 & 42.18 & 0.269 & 100 \\
\hline 45.26 & 0.252 & 69 & 45.37 & 0.251 & 75 \\
\hline 51.97 & 0.221 & 31 & 52.21 & 0.220 & 18 \\
\hline 63.67 & 0.184 & 39 & 63.48 & 0.184 & 63 \\
\hline 69.58 & 0.170 & 53 & 69.89 & 0.169 & 63 \\
\hline 74.37 & 0.160 & 11 & 74.46 & 0.160 & 13 \\
\hline 81.27 & 0.149 & 28 & 81.03 & 0.149 & 50 \\
\hline 83.56 & 0.145 & 36 & 83.76 & 0.145 & 50 \\
\hline 91.31 & 0.135 & 2 & 91.62 & 0.135 & 3 \\
\hline 94.60 & 0.132 & 12 & 95.28 & 0.131 & 18 \\
\hline 100.33 & 0.126 & 6 & 100.40 & 0.126 & 13 \\
\hline 104.15 & 0.123 & 1 & 103.81 & 0.123 & 3 \\
\hline 108.70 & 0.119 & 5 & 108.87 & 0.119 & 8 \\
\hline 112.40 & 0.117 & 5 & 113.13 & 0.116 & 5 \\
\hline 115.98 & 0.114 & 8 & 116.24 & 0.114 & 13 \\
\hline 122.46 & 0.110 & 10 & 123.29 & 0.110 & 10 \\
\hline 132.50 & 0.106 & 12 & 131.91 & 0.106 & 8 \\
\hline & & & & 8 \\
\hline
\end{tabular}

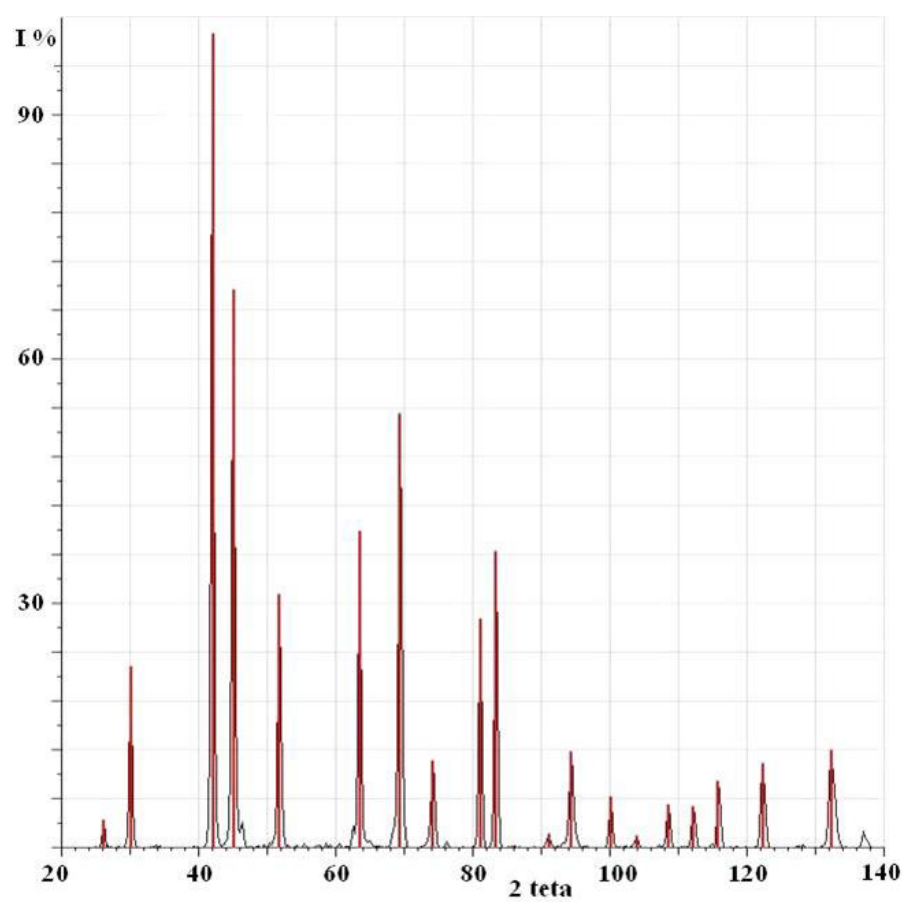

Fig. 4. Roentgendiffractogram «comparison-sample $2 »\left(\alpha-\mathrm{Fe}_{2} \mathrm{O}_{3}\right.$ in massive state) and comparison table of interplanar spacing $(\mathrm{d}, \mathrm{nm})$ of reflexes in the roentgenogram. 


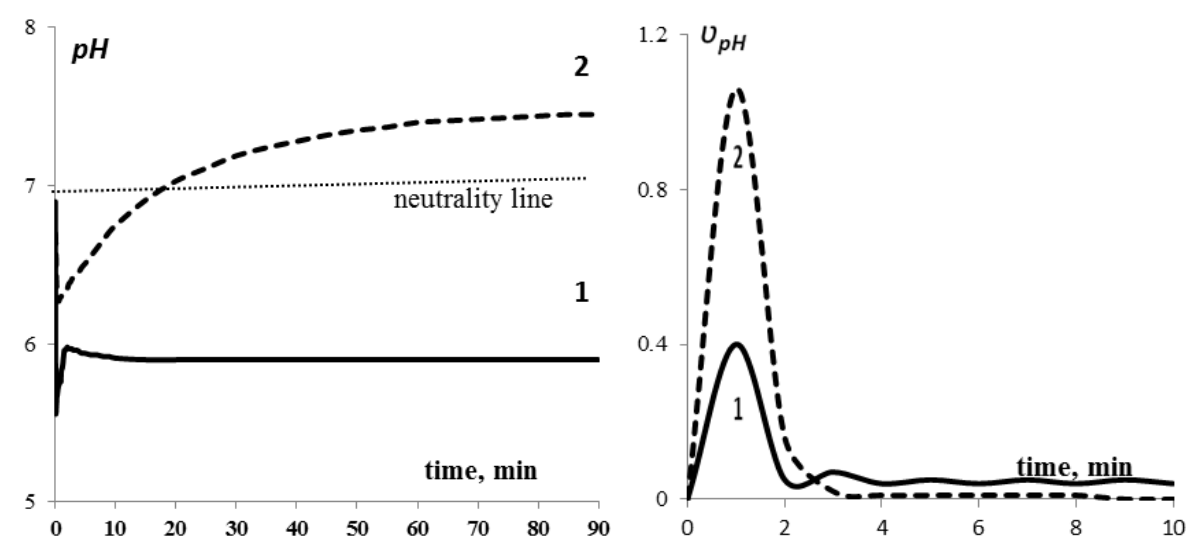

Fig.5. Kinetic curves of mass transfer variation $(\mathrm{pH})$ and speed instability $(\mathrm{v} \mathrm{pH})$ for the samples under study:

1) - «sample 1» (nanometric EEW iron)

2) - «comparison-sample 2» (ferric oxide with $\alpha-\mathrm{Fe}_{2} \mathrm{O}_{3}$ structure)

The sample behavior in the process of hydrolytic adsorption is different when ferric oxide of another structure is formed. As an example, the variation in speed gain and drop of the mass transfer for $\mathrm{Fe}_{3} \mathrm{O}_{4}$ sample in massive state is presented (Fig. 6). Fast speed gain and slow speed drop of moistening is typical for this oxide. This oxide is characterized as hydrophilic. Moistening process occurs in accordance with external diffusion inhibition [13-19]. The causes of different behavior of haematite and magnetite during hydrolytic adsorption are thoroughly described in works [5-7].

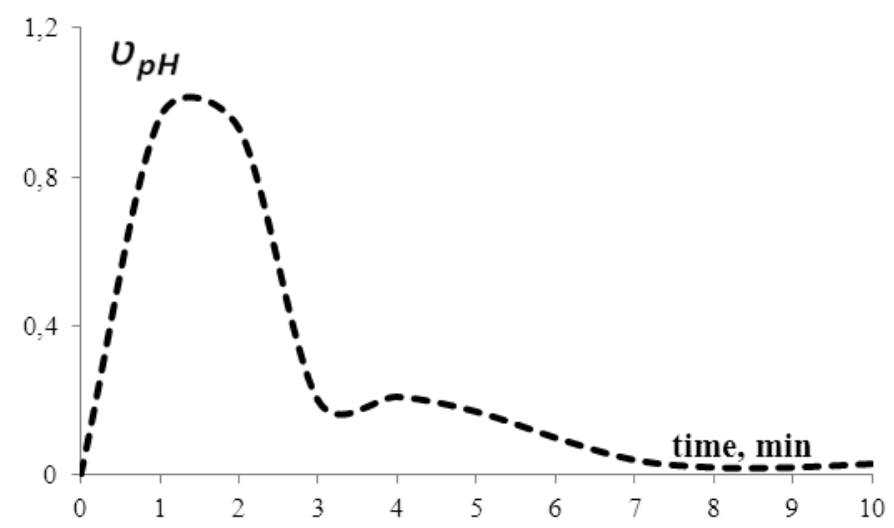

Fig.6. Kinetic curve of speed instability of mass transfer for ferric oxide with $\mathrm{Fe}_{3} \mathrm{O}_{4}$ structure.

Thus, the kinetic variation of the hydrolytic adsorption method allows us to give qualitative assessment of moistening of finely dispersed powders (hydrophilic behavior/ hydrophobic behavior). The diagnostic of moistening is based on the difference between speed gain and drop of moistening. The $\mathrm{pH}$ variations of aqueous suspension in a unit time is used as analytic moistening speed signal. Speed drop of moistening for $\alpha-\mathrm{Fe}_{2} \mathrm{O}_{3}$ haematite is fast which is caused by the processes of external diffusion inhibition and characterizes the hydrophobic behavior of surface. The speed gain of mass transfer for $\mathrm{Fe}_{3} \mathrm{O}_{4}$ magnetite is abrupt and its drop is slow and descrete which is caused by the processes of internal diffusion inhibition and characterizes the hydrophilic behavior of surface. The material of this article can be useful for its implementation in industrial, research and school laboratories. 


\section{Conclusions}

It was shown, that using the findings of investigation of hudrolytic adsorption the moistening of low-dimension particles can be characterized qualitatively as «hydrophilic behavior/ hydrophobic behavior». Since the surface moistening is predetermined by the set of acid-base centers typical for each structure the hudrolytic adsorption process for solid body with the same structure is identical. The use of $\mathrm{pH}$-metry results simplifies and accelerates the diagnostic process of surface moistening, which is economically viable and cost effective.

\section{References}

[1] P. A. Kouzov, L. Ya. Skryabina, Methods of physicochemical property definition of industrial dusts (L.: Chemistry, 1983).

[2] A. V. Korshunov, Dimensional and structural effects in the oxidation processes of metals (Tomsk: Publishing House TPU, 2013).

[3] A. I. Gusev, Nanomaterials, nanostructures, nanotechnologies (M.: FIZMATLIT, 2009)

[4] M. I. Lerner, V. V. Shamanskii, G. G. Savelyev, Bulletin of Tomsk Polytechnic University, V. 310. 2. (Tomsk: Publishing House TPU, 2007) pp 132-136

[5] K. V. Ikonnikova, L. F. Ikonnikova, E. A. Koltunova, Fundamental Research J., 10, 2. pp 2134-2137 (2015)

[6] K. V. Ikonnikova, L. F. Ikonnikova, E. A. Koltunova, Procedia Chemistry, 15. pp 167-173 (2015)

[7] K. V. Ikonnikova, L. F. Ikonnikova, E. A. Koltunova, Key Engineering Materials: Scientific Journal 683. pp 301-305 (2016)

[8] A. A. Zhigal'skii, L. F. Ikonnikova, T. S. Minakova, V. A. Mukhachev, P. E. Troyan, Russian Physics Journal, 39. iss. 6. pp 576-578 (1996)

[9] M. I. Lerner, N. V. Svarovskaya, S. G. Psakhe, O. V. Bakina, Russian nanotechnologies, 4. 9, pp 6-18 (2009)

[10] M. Lerner, A. Vorozhtsov, Sh. Guseinov, P. Storozhenko, Metal Nanopowders: Production, Characterization and Energetic Applications p 440 (2014)

[11] K. V. Ikonnikova, L. F. Ikonnikova, Yu. S. Sarkisov, T. S. Minakova, Theory and practice of pH-metry definition of acid-base properties of solid body surface. (Tomsk: Publishing House TPU, 2011) pp 99

[12] L. F. Ikonnikova, K. V. Ikonnikova, E. A. Koltunova, MATEC Web of Conferences (The 2nd International Youth Forum "Smart Grids", 2014) 19. 01017 p 3

[13] T. V. Kuznetsova, I. V. Kudryashov, V. V. Timashev, Physical chemistry of cementitious materials: Textbook for Chem-primary process. specialist. universities. (Moscow: high school, 1989) p 384

[14] S. C. McBain, H. H. Yiu, J. L. Dobson, Journal Nanomedicine. 3 (2) pp 169-180 (2008)

[15] R. M. Cornell, U. Schwertmann, The Iron Oxides: Structure, properties, reactions, occurences and uses. Second edition. Weinheim, Willey-VCH Verlag GmbH \& Co. KGaA p 683 (2003)

[16] E. N. Naumova, A. Y. Kalinkov, A. I. Kostrzhitsky, Refrigeration Tehnika $i$ Tehnologiya 5 (74) pp 46-51 (2001)

[17] S. P. Gubin, Yu. A. Koksharov, G. B. Chomutov, G. Y. Jurkov, Chemistry successes Journal 74 (6) pp 539-574 (2005)

[18] A. I. Kostrzhitsky, T. V. Cheban, R. A. Podolyan, Electronic processing of materials, 3 pp 50-55 (2007)

[19] I. S. Lyubutin, S. S. Starchikov, J. L. Dubinskaya, C.-R. Lin, V-Russia Conference on Nanomaterials, Nano-2013 Zvenigorod pp 314 (2013) 